\title{
Involvement of calmodulin and actin in directed differentiation of rat cortical neural stem cells into neurons
}

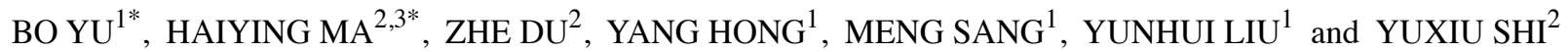 \\ ${ }^{1}$ Department of Neurosurgery, Shengjing Hospital Affiliated of China Medical University, Shenyang; ${ }^{2}$ Department of \\ Histology and Embryology, Institute of Pathology and Pathophysiology, China Medical University, Shenyang; \\ ${ }^{3}$ Department of Histology and Embryology, Dalian Medical University, Dalian, P.R. China
}

Received April 28, 2011; Accepted June 9, 2011

DOI: $10.3892 / \mathrm{ijmm} .2011 .734$

\begin{abstract}
Calmodulin (CaM) is a multifunctional receptor of intercellular $\mathrm{Ca}^{2+}$, which under different physiological conditions or at different developmental stages plays different roles in different tissues and cells. This study aimed to investigate the involvement of spatial expression and coexistence of CaM and actin in directed differentiation of rat cortical neural stem cells (NSCs) into neurons. Immunohistochemistry, RT-PCR and immunofluorescence dual-labeling technology was conducted to investigate the temporal and spatial pattern of $\mathrm{CaM}$ and actin proteins in neuron-oriented cortical NSC differentiation. Confocal laser scanning microscopy (CLSM) was used to observe changes of the coexistence in protein expression. Our results showed that cortical NSCs expressed and showed localized $\mathrm{CaM}$ and actin in a well-defined temporal order. In the process of rat neuron-oriented cerebral cortical NSC differentiation, CaM displayed a similar expression pattern with actin in the development of neurons, that is, both proteins extended into neurites following the sprouting and growth of neurons. These results suggest that the temporal and spatial pattern of CaM and actin expression is comparable to the growth of cell processes in differentiating NSCs. Therefore, both CaM and actin may jointly participate in the development and maturation of neurites, and this provides a theoretical basis for further study of the biological features of neuron-oriented NSC differentiation.
\end{abstract}

\section{Introduction}

As a multi-functional receptor of intercellular $\mathrm{Ca}^{2+}$, calmodulin (CaM) mediates many physiological processes regulated by

Correspondence to: Dr Yuxiu Shi, Department of Histology and Embryology, China Medical University, 92 Beima Road, Heping District, Shenyang, P.R. China

E-mail: yuxiushi@163.com

*Contributed equally

Key words: calmodulin, actin, neural stem cells, directed differentiation, neuron
$\mathrm{Ca}^{2+}$. $\mathrm{CaM}$ is extensively localized in almost all tissues of vertebrates, plants and prokaryotes. This protein is especially rich in the brain and some endocrine organs (1). CaM has been shown to play different roles in different tissues and cells, under different physiological conditions or at different developmental stages. In addition, actin is a ubiquitous cytoskeletal protein, which is mainly involved in the formation of microfilaments and maintains normal cellular functions with the assistance of intermediate filaments and microtubules (2-4). As a cytoskeletal protein, actin plays an important role in cell growth and development, which not only closely correlates with cell morphology, but also participates in multiple functions such as cell movement, substance transport, as well as cell division, differentiation and gene expression $(3,5)$.

It has been shown that alteration of the intracellular $\mathrm{Ca}^{2+}$ concentration is closely related to the function of actin (6). Therefore, $\mathrm{CaM}, \mathrm{a} \mathrm{Ca}^{2+}$ receptor should also affect the function of actin (7). However, it is not clear whether this correlation exists in the directed differentiation of rat cerebral cortical neural stem cells (NSCs) into neurons. In this study, immunohistochemistry, RT-PCR and image analysis technology were conducted to detect the spatial expression of $\mathrm{CaM}$ and its action during the process of rat neuron-oriented cerebral cortical NSC differentiation. Immunofluorescence dual-labeling technology was used to label CaM and actin. Confocal laser scanning microscopy (CLSM) was used to observe whether there is coexistence in the protein expression and correlation between CaM and actin. The main objective of this study was to provide the theoretical basis for further study of the biological characteristics of neuron-oriented directed differentiation of NSCs.

\section{Materials and methods}

Experimental animals. Wistar rats born within $24 \mathrm{~h}$ were provided by the Department of Laboratory Animals of the China Medical University. All procedures were approved by the Institutional Animal Care and Use Committee of the China Medical University, Shenyang, China.

Culture and differentiation of NSCs. Cerebral cortices from neonatal rats were taken out in a sterilized environment, placed in Hank's solution, rinsed, cut into small pieces and 
transferred into a centrifuge tube; $0.25 \%$ trypsin was added followed by incubation at $37^{\circ} \mathrm{C}$ for 25 min to digest; then DMEM/F12 serum-free medium was added to terminate the digestion. Mechanical force was used to disperse the cells which were then filtered by 4 layers of sterile gauze; cells were then centrifuged at $1,600 \mathrm{rpm} / \mathrm{min}$ for $5 \mathrm{~min}$; the supernatant was discarded and DMEM/F12 serum-free medium containing $20 \mathrm{ng} / \mathrm{ml} \mathrm{bFGF,} 20 \mathrm{ng} / \mathrm{ml} \mathrm{EGF,} 1 \% \mathrm{~N}_{2}$ supplement (all from Gibco, USA), 10,000 U/ml penicillin, 10,000 U/ml streptomycin (North China Pharmaceutical Company, Ltd., China) and $0.0014 \mathrm{ml} / 1$ diflucan (Pfizer, France) was added. Trypan blue staining was used to evaluate cell viability and the cell density in suspension was adjusted to $1-5 \times 10^{6} / \mathrm{ml}$, plated in $75 \mathrm{ml}$ culture flasks and placed at $37^{\circ} \mathrm{C}$ in a $5 \% \mathrm{CO}_{2}$ incubator. The culture medium was changed by half every two days. The cell morphology was observed using an inverted phase contrast microscope and the cell images were captured. Neurospheres formed after 7 days of primary culture with culture medium were collected into a centrifuge tube, centrifuged at $400 \mathrm{rpm}$ for $5 \mathrm{~min}$ and resuspended into a single cell suspension by the above culture medium. Cells were again plated into culture flasks at the same density. Cells were passaged for a total of 4 generations. To induce differentiation of NSCs, a single cell suspension of NSCs was plated onto poly-L-ornithine coated dishes at a density of $2 \times 10^{4}$ cell $/ \mathrm{ml}$ in the basic neurosphere medium with $10 \%$ FCS and $2 \mathrm{ng} / \mathrm{ml}$ BDNF and cultured for 7 days.

Histological analysis. Immunohistochemistry was performed to identify NSCs and neuron-oriented differentiation of NSCs. After NSCs were cultured for 7 days, the actively growing neurosphere suspension was dropped onto polylysine-treated coverslips and air-dried. To assess the expression of $\mathrm{CaM}$ and actin, the coverslips were taken out of culture capsules on Day 1,3,5 and 7 of the differentiation. Cells were then fixed by $4 \%$ paraformaldehyde for $20 \mathrm{~min}$, washed with PBS solution containing $0.03 \%$ Triton $\mathrm{X}-100$ for $10 \mathrm{~min}$, rinsed by PBS again, immersed in $3 \% \mathrm{H}_{2} \mathrm{O}_{2}$ for 10 min, washed with PBS, blocked by normal goat serum and incubated at $4^{\circ} \mathrm{C}$ overnight with one of the following primary antibodies: rabbit anti-rat nestin polyclonal antibody (1:200, Boster, Wuhan, China) for NSCs, rabbit anti-rat neuron-specific enolase (NSE) polyclonal antibody (1:400, Boster) for neurons, anti-rat CaM monoclonal antibody (1:2,000, Sigma, USA) and rabbit anti-rat actin polyclonal antibody (1:100, Boster). Then, the biotinylated secondary antibody and $\mathrm{ABC}$ complex (Boster) were added in sequence followed by development with DAB. The reaction time was controlled under a microscope. Samples were washed again by distilled water, dehydrated by series of alcohols, processed by xylene and sealed by Balsam neutral. The control group used PBS to replace the primary antibody with all other staining steps same as above. The Olympus optical microscope was used to observe the cells and to capture images.

Immunofluorescence double-labeling of CaM and actin. Coverslips were taken out from the culture dishes on Day 1, 3,5 and 7 of differentiation, fixed with $4 \%$ paraformaldehyde for $20 \mathrm{~min}$, washed with PBS solution containing $0.03 \%$ Triton X-100, rinsed with PBS, blocked with normal goat serum and incubated with CaM $(1: 2,000)$ and actin $(1: 300)$
Table I. The primers of calmodulin $(\mathrm{CaM})$ and $\beta$-actin.

\begin{tabular}{llc}
\hline Name & Primers & $\begin{array}{c}\text { Product } \\
\text { size (bp) }\end{array}$ \\
\hline CaM & $\begin{array}{l}\text { Forward 5'-ggcatcetgctttagcctgag-3' } \\
\text { Reverse 5'-acatgctatccctctcgtgtga-3' }\end{array}$ & 328 \\
3-actin & $\begin{array}{l}\text { Forward 5'-atcacccacactgtgcccatc-3' } \\
\text { Reverse 5'-acagagtacttgcgctcagga-3' }\end{array}$ & 542 \\
\hline
\end{tabular}

mixed antibodies at $4{ }^{\circ} \mathrm{C}$ overnight, followed by addition of Cy3-labeled anti-mouse IgG antibody (1:100, Gibco) and FITClabeled anti-rabbit IgG antibody (1:50, Boster) mixed secondary antibodies for $1 \mathrm{~h}$, rinsed by PBS and sealed by $50 \%$ glycerol. Fluorescent signals were detected using a Nikon compound fluorescent microscope and a Nikon C1 Plus confocal microscope (Nikon, Japan).

Reverse transcription-polymerase chain reaction to detect CaM. Total-mRNA was extracted from differentiation of NSCs according to the instructions of the TRIzol kit. The primers designed by Shenggong Biotech Company (Shanghai, China) according to the serial numbers from GenBank are shown in Table I. PCR was performed using Taq Dynazyme (Takara, Dalian, China) under standard conditions. The reaction was started at $94^{\circ} \mathrm{C}$ for 4 min and amplification for $\mathrm{CaM}$ occured for 32 cycles of $94^{\circ} \mathrm{C}$ for $30 \mathrm{sec}, 58^{\circ} \mathrm{C}$ for $30 \mathrm{sec}, 72^{\circ} \mathrm{C}$ for $40 \mathrm{sec}$, followed by $7 \mathrm{~min}$ final extension at $72^{\circ} \mathrm{C}$. $\beta$-actin mRNA was co-amplified with CaM mRNA as an internal control. The products were observed after electrophoresis on $1.2 \%$ agarose gel, and the density of each band was analyzed on the Gel Image Analysis System. The level of CaM mRNA was determined by calculating the density ratio of $\mathrm{CaM}$ mRNA/ $\beta$-actin mRNA.

Statistical analysis. The positive products of $\mathrm{CaM}$ and actin in directed differentiation of NSCs into neurons were analyzed with the Motic Instruments Images Advanced 3.0 image analysis system. Under the microscope (x400), 50 cells were quantified in each group, and the average integral optical density (IOD) was determined. Differences between mean values of $\mathrm{CaM}$ or actin for different time points were analyzed using one-way ANOVA, and independent sample t-test was performed to detect the difference between mean values of $\mathrm{CaM}$ and actin at the same time points. The data are expressed as mean $\pm \mathrm{SD}$ and the difference was considered significant at $\mathrm{P}<0.05$.

\section{Results}

Identification of NSCs and directed differentiation of NSCs into neuron. On Day 7 of culture, each colony included up to dozens to hundreds of cells. Colony forming units appeared as spherical, oval or morular shapes and grew in suspension (Fig. 1A) with good vitality. These cell colonies showed strong immunological reaction signals by nestin immunohistochemical staining (Fig. 1B).

NSCs were dispersed by mechanical force and cultured in medium containing serum and induction factors. After $4 \mathrm{~h}$, 

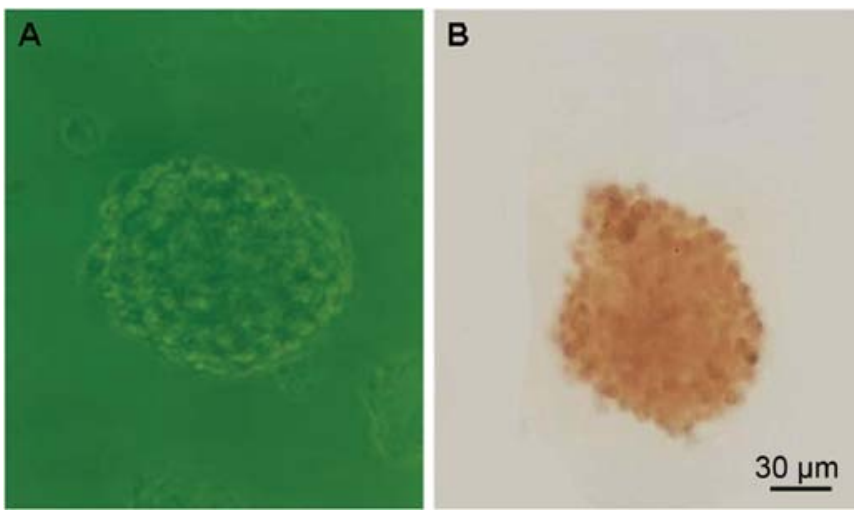

Figure 1. Culture and identification of neonatal rat cortical NSCs. (A) NSCs proliferating into neurospheres. (B) Immunohistochemical staining showed that neurospheres were nestin positive.
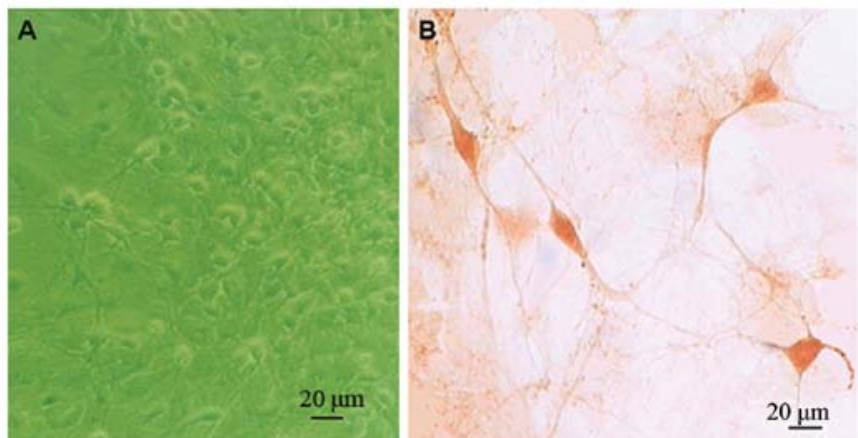

Figure 2. Differentiation of neonatal rat cortical NSCs. (A) On Day 7 of differentiation of neonatal rat cortical NSCs, various morphological cells were observed under inverted phase contrast microscopy. (B) Immunohistochemical staining showed that some of these differentiating cells were NSE-positive, which were part of the directed differentiation of neonatal rat cortical NSCs into neurons.

cells started to attach and some cells differentiated to possess neurites on Day 2. Among these differentiated cells, there were three types: some were larger cells, attached flat on the coverslip, with many thick neurites; these cells had a large quantity. The second type of cells were smaller, with fewer but very long neurites, highly refractive and a part of which sat upon the larger cells; there was a small quantity of these cells. The last type of cells were the smallest and fewest, with relatively short and thin neurites (Fig. 2A). Immunohistochemical staining showed that some of these directed NSCs were NSE-positive (Fig. 2B).

CaM immunocytochemical staining. During the directed differentiation of cerebral cortical NSCs into neurons, on the first day, cell bodies were small, and neurites were short. CaM proteins were mainly located in and around nucleus (Fig. 3A). On the Days 3, 5 and 7 of differentiation, as the cells grew, cell bodies became bigger, and neurites became longer and more plentiful. A positive expression of CaM proteins was observed in both the cytoplasm and in neurites, which increased as the neurites extended. We could see segmental positive expression products extending to the extremities of the neurites. However, the positive reaction of the nucleus was weaker than that on the
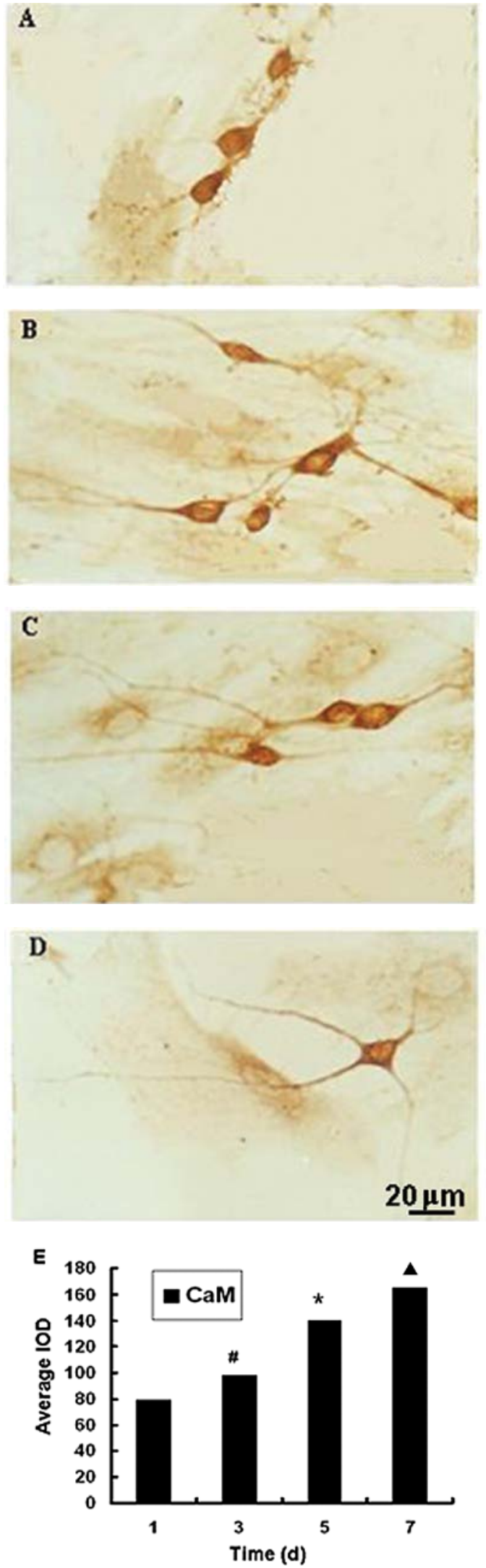

Figure 3. CaM immunohistochemical staining during the directed differentiation of neonatal rat cortical NSCs into neurons. (A) At the early stage of differentiation, cell bodies were small, and a brown positive reaction was shown in and around the nucleus. (B-D) As cells differentiated, positive reaction products extended segmentally into the cytoplasm and neurites. (E) Average integral optical density value of $\mathrm{CaM}$ during different stages of the directed differentiation of cerebral cortical neural stem cells into neurons. The data are expressed as mean $\pm \mathrm{SD},{ }^{\#} \mathrm{P}<0.01$ vs. Day $1 ;{ }^{*} \mathrm{P}<0.01$ vs. Day 3; $\bullet \mathrm{P}<0.01$ vs. Day 5 . 

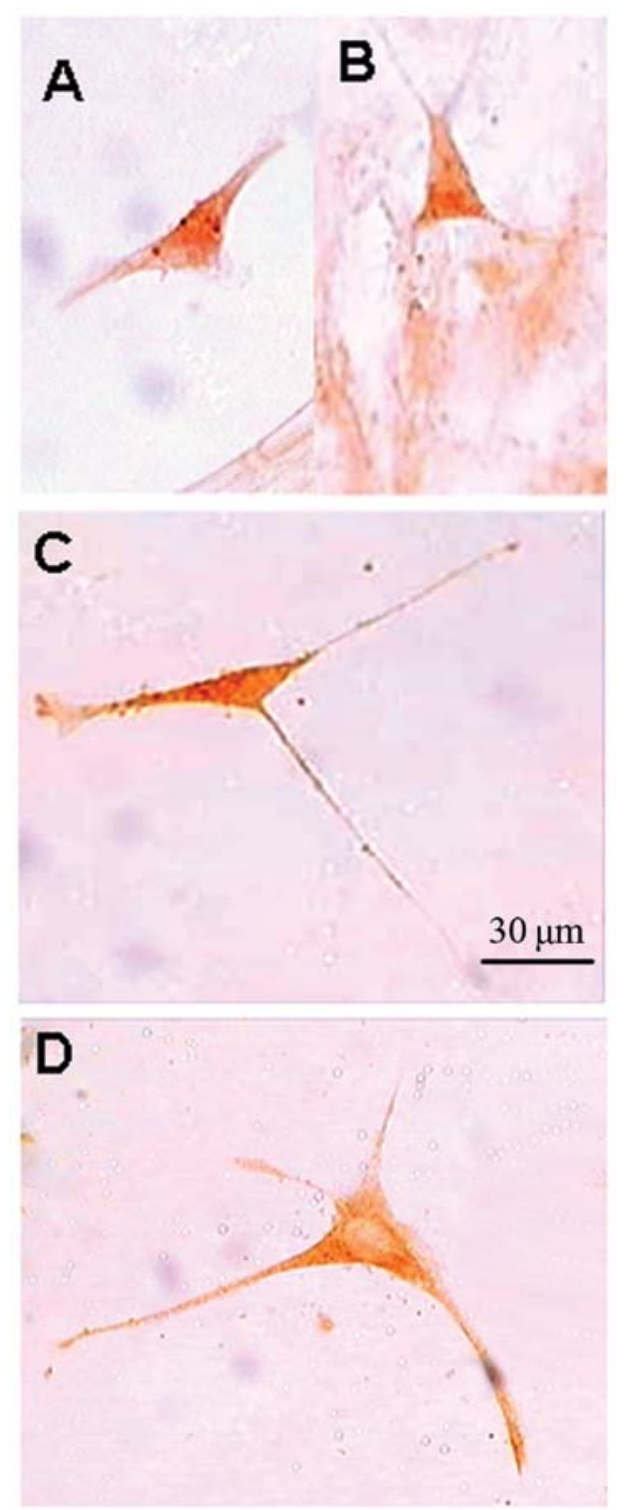

E

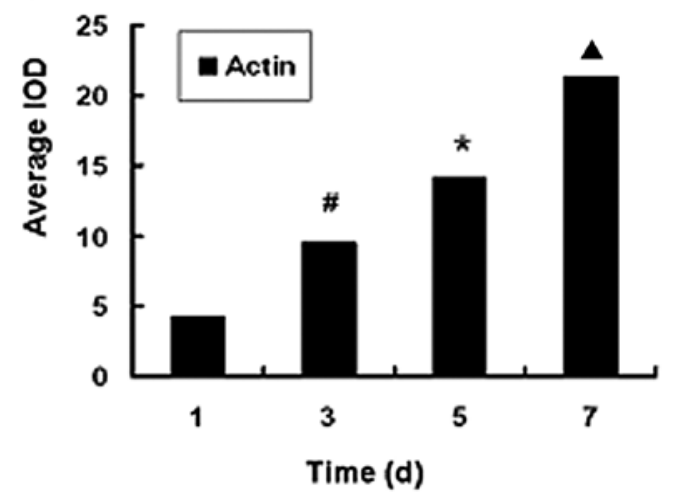

Figure 4. Actin immunohistochemical staining during the directed differentiation of neonatal rat cortical NSCs into neurons. (A) On the first day of differentiation, actin was mainly expressed around the nucleus and pinosomes were seen in the cytoplasm. (B and C) On Day 3 and 5 of differentiation, actin was expressed both in the cytoplasm and in neurites. Byssoid brown actin positive products could be seen. (D) On Day 7 of differentiation, filiform cytoskeleton was seen clearly in cytoplasm and neurites, which was arranged in parallel to the neurites and extended with them. (E) The average integral optical density value of actin during the different stages of directional differentiation of cerebral cortical NSCs into neurons. The data are expressed as the mean $\pm \mathrm{SD} ;{ }^{*} \mathrm{P}<0.01$ vs. Day $1 ;{ }^{*} \mathrm{P}<0.01$ vs. Day $3 ;{ }^{\wedge} \mathrm{P}<0.01$ vs. Day 5.
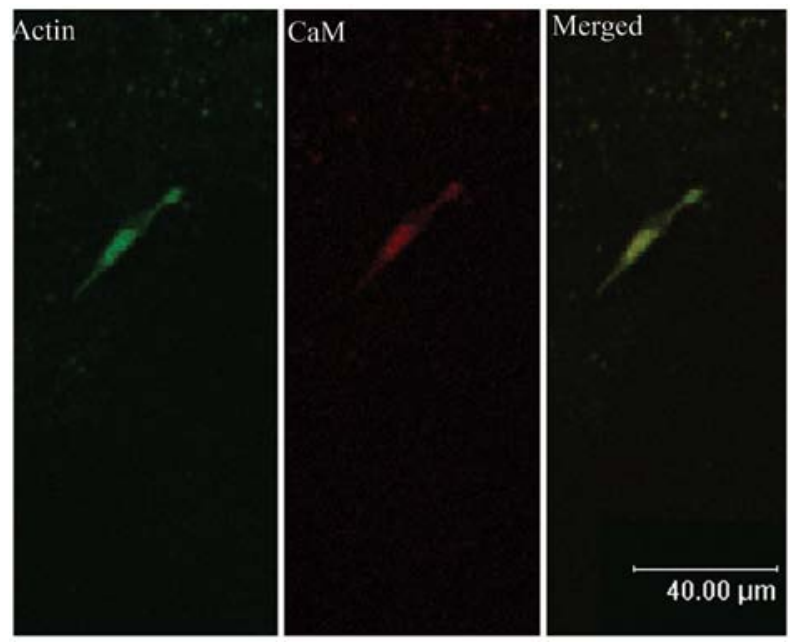

Figure 5. Observation of fluorescence double-labeled $\mathrm{CaM}$ and actin on the first day of differentiation. A smaller cell body with short neurites was observed (actin, green; CaM, red; merged image, yellow). Distribution patterns of CaM and actin were largely similar.

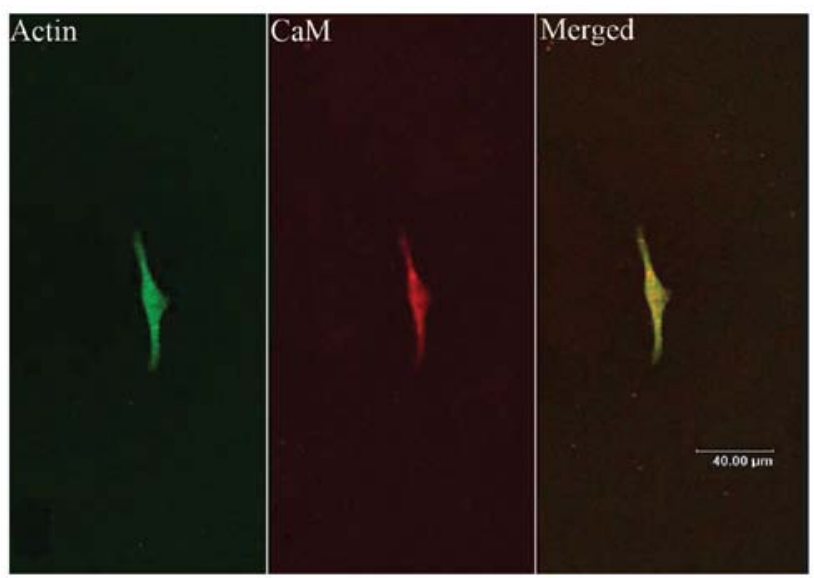

Figure 6. Observation of fluorescence double-labeled CaM and actin on Day 3 of differentiation. Cell bodies were enlarged and neurites were elongated. $\mathrm{CaM}$ and actin had similar distribution patterns and gradually extended into the neurites. Orange fluorescence appeared around the nucleus.

first day (Fig. 3B-D). The average integral optical density value of CaM during the different stages of directed differentiation of cerebral cortical NSCs into neurons is shown in Fig. 3E.

Actin immunocytochemical staining. Actin positive staining shown as brown granulations were located in the cytoplasm of neurons, especially around the nucleus. However, there was no expression of actin in the nucleus. On the first day after differentiation, actin was expressed mainly around the nucleus, and pinosomes were seen in the cytoplasm (Fig. 4A). However, with maturation of cells, on Day 3 and 5, actin was expressed both in the cytoplasm and in neurites. It was more strongly expressed around the nucleus, and its distribution near the base of neurites appeared to be reticular (Fig. 4B). On Day 7 after differentiation, cells were nearly mature. The expression of actin was even more increased around the nucleus, and the brown positive reaction was darker. The filiform cytoskeleton was arranged in parallell in the cytoplasm and extended into 


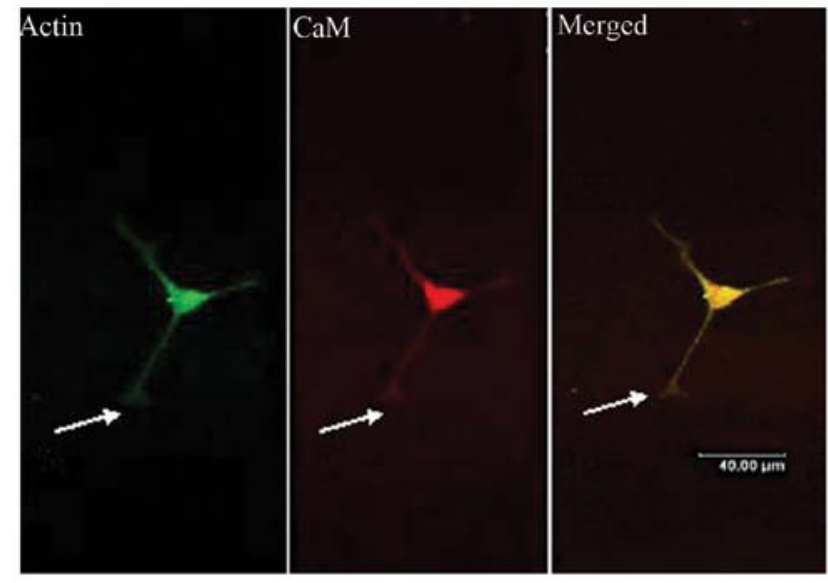

Figure 7. Observation of fluorescence double-labeled CaM and actin on Day 5 of differentiation. Cell bodies further enlarged and neurites elongated in continuously differentiating neurons. Expression of $\mathrm{CaM}$ and actin extended like a duck web into the extremities of neurites.

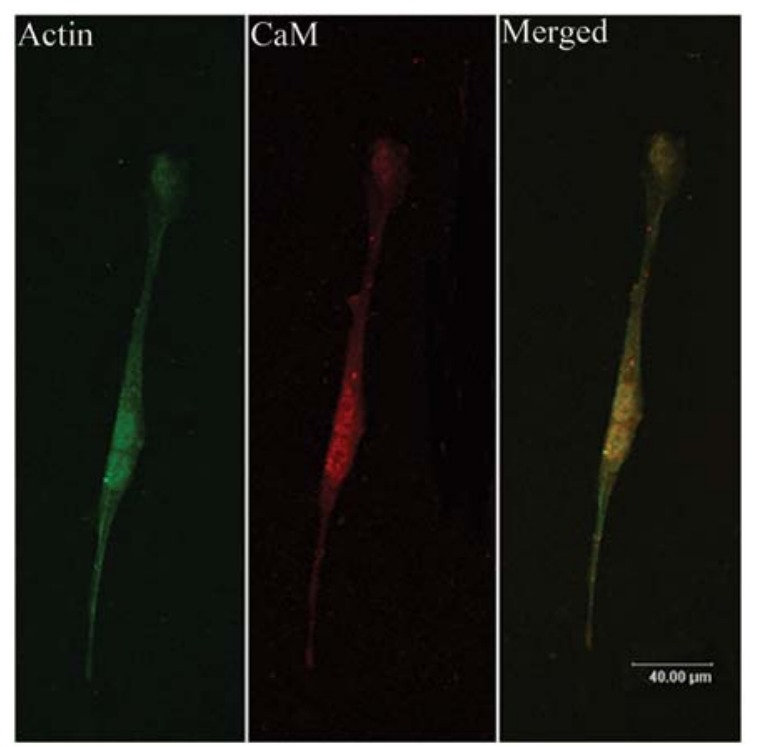

Figure 8. Observation of fluorescence double-labeled $\mathrm{CaM}$ and actin on Day 7 of differentiation. Orange fluorescence appeared both around the nucleus and at the neurite terminus. However, they also showed independent existence without overlapping within part of the process.

neurites (Fig. 4C and D). On Days 1, 3, 5 and 7, the quantity of expression gradually increased, and there were significant differences in actin protein expression $(\mathrm{P}<0.01)$ (Fig. 4E).

Co-expression of CaM and actin in differentiating neurons. In the same sample, excitation by different wavelengths of monofluorescence can reveal the existence and distribution pattern of red fluorescent CaM (excitation wavelength of $543 \mathrm{~nm}$ ) and green fluorescent actin (excitation wavelength of $488 \mathrm{~nm}$ ) in neurons. They showed a similar localization distribution. Specifically, at the early stage of differentiation, these two proteins concentrated inside the nucleus and at the vicinity of the nucleus (Fig. 5). With the maturation of neurons, the fluorescence gradually extended into the cytoplasm and neurites of the continuously differentiating neurons (Figs. 6-8). These two fluorescence signals largely co-localized in the cytoplasm
A

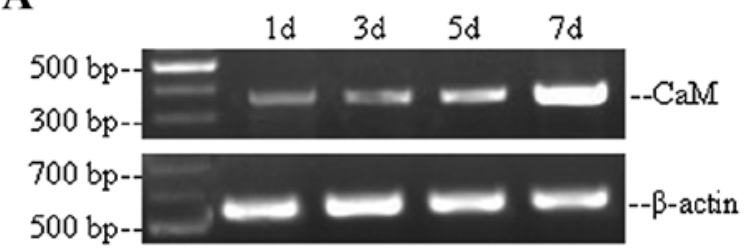

B

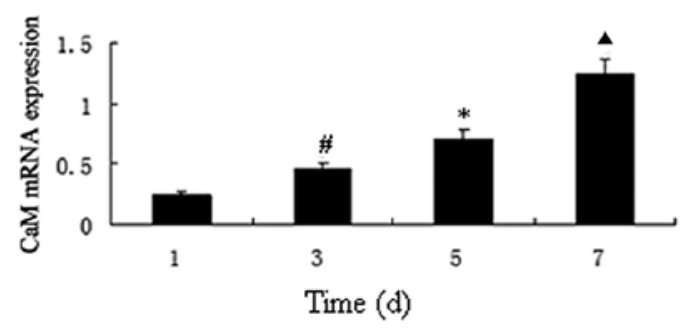

Figure 9. Expression of CaM mRNA. (A) The representative gel pattern of $\mathrm{CaM}$ and $\beta$-actin of cDNA bands. (B) Densitometric analysis of $\mathrm{CaM}$ of the semi-quantitative RT-PCR. Data are expressed as the mean \pm SD ${ }^{\#} \mathrm{P}<0.05$ vs. Day $1 ;{ }^{*} \mathrm{P}<0.05$ vs. Day $3 ;{ }^{\wedge} \mathrm{P}<0.05$ vs. Day 5.

and neurites and overlapped with each other. However, in some cases, they showed independent existence without overlapping, suggesting the correlation between $\mathrm{CaM}$ and actin.

Expression of CaM and actin $m R N A$. The representative gel pattern of CaM assessed the level of CaM mRNA normalized to the $\beta$-actin mRNA level. The level of CaM mRNA showed a significant up-regulation on Day 3,5 and 7 compared with that on the first day after differentiation (Fig. 9).

\section{Discussion}

NSCs are multipotent with the ability of self-renewal. They can differentiate into neurons, astrocytes and oligodendrocytes (8). Currently, the regular methodology is to use a combination of FGF-2 and EGF co-factors to culture NSCs in vitro (9-12). In this study, we used the same method to culture NSCs and confirmed the cell identify using immunohistochemical staining for nestin, a protein that disappears along with the differentiation and maturation of the neural epithelium (13-16).

$\mathrm{CaM}$ has been proven to be a versatile $\mathrm{Ca}^{2+}$-binding protein and exists in neural cells in a large quantity (17-19). In the nervous system, CaM participates in many important biological functions while multiple activities of neural cells are subject to the regulation by $\mathrm{Ca}^{2+}-\mathrm{CaM}$ signaling (17). Previous studies have proven that CaM plays important roles in many biological processes, such as early development of neurites, regulation of microtubule formation, neuron metabolism, axoplasmic transport, synaptic transmission and neurotransmitter synthesis, release and transport (20-23).

As a cytoskeletal protein, actin plays a key role in maintaining the mobility of the neuronal growth cones (24-27). Our results confirmed that in the process of neuron-oriented rat cerebral cortical NSC differentiation, the expressions of $\mathrm{CaM}$ and actin were in accordance with each other, they extended into the neurites and even in the duck-web like lamellipodia corresponding to the growth and development of 
neurons, which was shown both under CLSM and by immunohistochemistry. These results suggest that the role of actin in neurite development is modulated by $\mathrm{CaM}$. At the early stage of neuron-oriented cerebral cortical NSC differentiation, the relationship between $\mathrm{CaM}$ and actin was closely related to the robust neuron functional activities. At the early developmental stage of neural cells, an increase in intracellular free $\mathrm{Ca}^{2+}$ concentration affected the initiation of cell migration and the connection between cells, which maintained cell morphology of the neuron and promoted the extension of neurites. However, those functions executed by $\mathrm{Ca}^{2+}$ needed the involvement of $\mathrm{CaM}$ (28). In addition, $\mathrm{Ca}^{2+}$ was a very effective regulator in which it could significantly affect the structure and function of actin (29). $\mathrm{Ca}^{2+}-\mathrm{CaM}$ have been shown to be related to the assembly and disassembly of tubulin, thereby affecting the polymerization/depolymerization of microfilaments, the dynamic change and balance which are important regulatory factors for cell movement, attachment and the cell division cycle (28). Therefore, actin determined the active movement of lamellipodia and filopodia in neuronal growth cones subjected to the influence of $\mathrm{Ca}^{2+}-\mathrm{CaM}$, so that filopodia and lamellipodia were able to extent leading to the growth and extension of axons (30). This study only observed the co-expression of actin and $\mathrm{CaM}$ in the early stage of differentiating neural cells. However, how CaM affects the impact of actin on growth cones, as well as whether this similar expression pattern exists at late stages of neuronal differentiation, are still subject to study.

\section{Acknowledgements}

The project was supported by grants of the S. and T. Research Project of Education Bureau, Liaoning Province, China (Grant nos. 2008779, 2008851).

\section{References}

1. Kitamura Y,Arima T,Imaizumi R, Sato T and Nomura Y: Inhibition of constitutive nitric oxide synthase in the brain by pentamidine, a calmodulin antagonist. Eur J Pharmacol 289: 299-304, 1995.

2. Schaloske RH,Lusche DF, Bezares-Roder K, Happle K, Malchow D and Schlatterer $\mathrm{C}: \mathrm{Ca}^{2+}$ regulation in the absence of the iplA gene product in Dictyostelium discoideum. BMC Cell Biol 6: 13, 2005

3. Dabrowska R, Kulikova N and Gagola M: Nonmuscle caldesmon: its distribution and involvement in various cellular processes. Protoplasma 224: 1-13, 2004.

4. Levinson H, Moyer KE, Saggers GC and Ehrlich HP: Calmodulinmyosin light chain kinase inhibition changes fibroblast-populated collagen lattice contraction, cell migration, focal adhesion formation, and wound contraction. Wound Repair Regen 12: 505-511, 2004

5. Larsson C: Protein kinase $\mathrm{C}$ and the regulation of the actin cytoskeleton. Cell Signal 18: 276-284, 2006.

6. Furukawa R, Maselli A, Thomson SA, Lim RW, Stokes JV and Fechheimer M: Calcium regulation of actin crosslinking is important for function of the actin cytoskeleton in Dictyostelium. J Cell Sci 116: 187-196, 2003.

7. Lieto-Trivedi A and Coluccio LM: Calcium, nucleotide, and actin affect the interaction of mammalian Myolc with its light chain calmodulin. Biochemistry 47: 10218-10226, 2008.

8. Temple S and Alvarez BA: Stem cells in the adult mammalian central nervous system. Curr Opin Neurobiol 9: 135-141, 1999.
9. Gritti A, Coval L, Parati EA, Galli R and Vescovi AL: Basic fibroblast growth factor supports the proliferation of epidermal growth factor-generated neuron precursor cells of the adult mouse CNS. Neurosci Lett 185: 151-154, 1995.

10. Ciccolini $F$ and Svendsen $C N$ : Fibroblast growth factor 2 (FGF-2) promotes acquisition of epidermal growth factor (EGF) responsiveness in mouse striatal precursor cells: identification of neural precursors responding to both EGF and FGF-2. J Neurosci 18: 7869-7880, 1998

11. Takahashi T, Palmer TD and Gage FH: Retinoic acid and neurotrophins collaborate to regulate neurogenesis in adult-derived neural stem cell cultures. J Neurobiol 38: 65-81, 1999.

12. Tatebayashi T, Iqbal K and Grundke-Iqbal I: Dynamic regulation of expression and phosphorylation of tau by fibroblast growth factor-2 in neural progenitor cells adult rat hippocampus. J Neurosci 19: 5245-5254, 1999.

13. Pérez Castillo A, Aguilar-Morante D, Morales-García JA and Dorado J: Cancer stem cells and brain tumors. Clin Transl Oncol 10: 262-267, 2008

14. Gilyarov AV: Nestin in central nervous system cells. Neurosci Behav Physiol 38: 165-169, 2008.

15. Giliarov AV: Nestin in the cells of the central nervous system. Morfologiia 131: 85-90, 2007 (In Russian).

16. Daniel C, Albrecht H, Lüdke A and Hugo C: Nestin expression in repopulating mesangial cells promotes their proliferation. Lab Invest 88: 387-397, 2008.

17. Thomas R, Bill C and Debra B: Cellular signaling through multifunctional $\mathrm{Ca}^{2+} / \mathrm{Calmodulin}$-dependent protein kinase II. Biol Chem 276: 3719-3722, 2001.

18. Yamasaki TR, Blurton-Jones M and Morrissette DA: NSCs improve memory in an inducible mouse model of neuronal loss. J Neurosci 27: 11925-11933, 2007.

19. Jori FP, Napolitano MA, Melone MA, Cipollaro M, Cascino A, Altucci L, Peluso G, Giordano A and Galderisi U: Molecular pathways involved in neural in vitro differentiation of marrow stromal stem cells. J Cell Biochem 94: 645-655, 2005.

20. Deisseroth K, Heist EK and Tsien RW: Translocation of calmodulin to the nucleus supports CREB phosphorylation in hippocampal neurons. Nature 392: 198-202, 1998.

21. Corcoran EE and Means AR: Defining $\mathrm{Ca}^{2+} /$ calmodulin-dependent protein kinase cascades in transcriptional regulation. J Biol Chem 276: 2975-2978, 2001.

22. Laporte SA, Oakley RH and Caron MG: Signal transduction. Bringing channels closer to the action. Science 293: 62-63, 2001.

23. Kasahara J, Fukunaga K and Miyamoto E: Activation of calcium/ calmodulin-dependent protein kinase IV in long term potentiation in the rat hippocampal CAI region. J Biol Chem 276: 24044-24050, 2001.

24. Wang G, Krishnamurthy K, Chiang YW, Dasgupta S and Bieberich E: Regulation of neural progenitor cell motility by ceramide and potential implications for mouse brain development. J Neurochem 106: 718-733, 2008.

25. Dore-Duffy P, Katychev A, Wang X and Van Buren E: CNS microvascular pericytes exhibit multipotential stem cell activity. J Cereb Blood Flow Metab 26: 613-624, 2006.

26. Lo Iacono L and Gross C: Alpha- $\mathrm{Ca}^{2+} / \mathrm{calmodulin}^{2}$ dependent protein kinase II contributes to the developmental programming of anxiety in serotonin receptor 1A knock-out mice. J Neurosci 28: 6250-6257, 2008.

27. Wei J, Zhang M, Zhu Y and Wang JH: $\mathrm{Ca}(2+)$-calmodulin signalling pathway up-regulates GABA synaptic transmission through cytoskeleton-mediated mechanisms. Neuroscience 127: 637-647, 2004.

28. Nebl T and Fisher PR: Intracellular $\mathrm{Ca}^{2+}$ signals in Dictyostelium chemotaxis are mediated exclusively by $\mathrm{Ca}^{2+}$ influx. J Cell Sci 110: 2845-2853, 1997.

29. Zuo H, Cui B, She X and Wu M: Changes in Guinea pig cochlear hair cells after sound conditioning and noise exposure. J Occup Health 50: 373-379, 2008

30. Papp S, Szabo E, Kim H, McCulloch CA and Opas M: Kinasedependent adhesion to fibronectin: regulation by calreticulin. Exp Cell Res 314: 1313-1326, 2008. 